Brief report

\title{
Genetic polymorphisms of glutathione S-transferases GSTM1, GSTT1, GSTP1 and GSTA1 as risk factors for schizophrenia
}

\author{
Paolo Gravina $^{\text {a,c,* }}$, Ilaria Spoletini ${ }^{\text {b }}$, Silvia Masini ${ }^{\text {c }}$, Alessandra Valentini ${ }^{\text {a,c }}$, Diego Vanni ${ }^{\text {b }}$, \\ Eleonora Paladini ${ }^{c}$, Paola Bossù ${ }^{b}$, Carlo Caltagirone ${ }^{b}$, Giorgio Federici ${ }^{c}$, \\ Gianfranco Spalletta b,1, Sergio Bernardini c,1 \\ a Clinical Molecular Biology Laboratory, Dept. of Laboratory Medicine, PTV, Rome, Italy \\ ${ }^{\mathrm{b}}$ Istituto di Ricovero e Cura a Carattere Scientifico Fondazione Santa Lucia, Rome, Italy \\ c Department of Internal Medicine, Tor Vergata University, Rome, Italy
}

\section{A R T I C L E I N F O}

\section{Article history:}

Received 28 January 2009

Received in revised form 30 September 2010

Accepted 12 October 2010

\section{Keywords:}

Glutathione S-transferases

Schizophrenia

Reactive oxygen species

\begin{abstract}
A B S T R A C T
Oxidative damage is thought to play a role in the predisposition to schizophrenia. We determined if the polymorphisms of the GSTP1, GSTM1, GSTT1 and GSTA1 genes, which affect the activity of these enzymes against oxidative stress, have a role as susceptibility genes for schizophrenia, analyzing 138 schizophrenic patients and 133 healthy controls. We found that the combination of the absence of GSTM1 gene with the of the GSTM1 gene with the polymorphism GSTA1 ${ }^{*} B /{ }^{*} B$, and the presence of the GSTT1 gene, represents a risk factor for schizophrenia, indicating that the combination of different GST polymorphisms has a role in the predisposition to schizophrenia, probably affecting the capacity of the cell to detoxify the oxidized metabolites of catecholamines.
\end{abstract}

(c) 2010 Elsevier Ireland Ltd. All rights reserved.

\section{Introduction}

Schizophrenia is a neuropsychiatric disorder that affects approximately $1 \%$ of the population. (Freedman, 2003; Marini et al., 2008; Spoletini et al., 2008). One of the factors contributing to the development of schizophrenia is thought to be oxidative damage to neuronal cells. Glutathione S-transferases (GSTs) are phase II biotransformation enzymes involved in the detoxification of a wide range of compounds, including products generated by the oxidative damage (Valentini et al., 2008).

Some members of this family present well-established polymorphisms in the human population, affecting their expression and activity (Valentini et al., 2008). We decided to evaluate the presence of polymorphisms of four members of the GST family, GSTP1, GSTM1, GSTT1 and GSTA1, in schizophrenia.

\section{Materials and methods}

2.1. Subjects

One hundred thirty-eight consecutive patients with a diagnosis of schizophrenia according to the Diagnostic and Statistical Manual of Mental Disorders (DSM-IV)

* Corresponding author. Clinical Molecular Biology Laboratory, Dept. of Laboratory Medicine, PTV. Viale Oxford 81, 00133 Rome, Italy. Tel.: + 39 620902259; fax: + 39 620902357.

E-mail address: paolo.gravina@ptvonline.it (P. Gravina).

1 G.S and S.B. contributed equally to this work.
(American Psychiatric Association, 2004) were recruited from two outpatient clinics in central Italy. Detailed information on the diagnostic process is described elsewhere (Marini et al., 2008; Spoletini et al., 2008). Severity of schizophrenia symptoms was assessed by using the Positive and Negative Syndrome Scale (PANSS) (Kay et al., 1987). Exclusion criteria were as follows: history of alcohol or drug dependence or traumatic head injury; any past or present major medical or neurological illness; any additional psychiatric disorder; any brain pathology identified on magnetic resonance imaging (T2 or FLAIR scans); and mental retardation. All patients were receiving stable oral doses of one or more atypical antipsychotic drugs such as risperidone, quetiapine, and olanzapine. Antipsychotic dosages were converted to estimated equivalent dosages of olanzapine (Oquendo et al., 2003).

One hundred thirty-three healthy comparison (HC) subjects were recruited in the same geographic area and rigorously matched with the schizophrenic patients for age and gender (Table 1).

The study was approved by Santa Lucia Foundation Ethics Committee and undertaken in accordance with the principles laid down in the Declaration of Helsinki. A written consent was obtained from all participants after a full explanation of the procedures of the study.

\subsection{Experimental procedures}

Purification of DNA samples from peripheral blood samples and genotyping of GSTA1, GSTP1, GSTM1 and GSTT1 genes were performed as previously described (Bernardini et al., 2005; Gaziev et al., 2010).

\subsection{Statistical analysis}

Comparisons between the two groups were performed using $t$-tests and chi-square tests. Logistic regression analysis was performed to estimate odds ratios (OR) with $95 \%$ confidence intervals $(\mathrm{CI})$ and the combined effects of GST genotypes.

All these tests were two-tailed, and the level of statistical significance was defined as $p \leq 0.05$. 
Table 1

Sociodemographic and clinical characteristics of 138 patients with schizophrenia and 133 healthy comparison subjects.

\begin{tabular}{|c|c|c|c|c|c|}
\hline Characteristics & Schizophrenic patients $(N=138)$ & Comparison subjects $(N=133)$ & $t$ & $d f$ & $p$ \\
\hline Age (years)* & $47.33 \pm 5.18$ & $47.84 \pm 3.80$ & -.110 & 88 & 0.9127 \\
\hline Age at the onset of the illness (years)* & $23.80 \pm 8.23$ & - & - & - & - \\
\hline Duration of the illness (years)* & $19.27 \pm 10.62$ & - & - & - & - \\
\hline Olanzapine Equivalents (mg/day)* & $26.70 \pm 29.58$ & - & - & - & - \\
\hline Gender (male) ${ }^{* *}$ & $82(60 \%)$ & $62(47 \%)$ & 2.997 & 2 & 0.2235 \\
\hline
\end{tabular}

$\mathrm{SD}=$ standard deviation; $d f=$ degrees of freedom.

$*=$ mean $\pm S D$

$* *=N(\%)$.

Statistical analysis was carried out with the SPSS statistical package version 15.0 and the Sample Power package version 2.0.

\section{Results}

The genotype distribution among controls was in Hardy-Weinberg equilibrium for GSTA1 (chi-square $=2.947 ; d f=1 ; p=0.09$ ) and GSTP1 (chi-square $=0.361 ; d f=1 ; p=0.55$ for Ile105Val polymorphism; and chi-square $=0.247 ; d f=1 ; p=0.62$ for Ala114Val polymorphism). The Hardy-Weinberg equilibrium test was not applied to GSTM1 and GSTT1 genotypes, because multiplex PCR assay cannot discriminate the heterozygous presence of the allele from the homozygous presence. However, the distributions of these two genotypes were similar to those reported in other studies (Cornelis et al., 2007).

We did not find significant differences in the two groups for the polymorphisms analyzed, although we found a higher frequency of the presence of the GSTT1 gene and the GSTM1 null and the GSTA1 ${ }^{*} B{ }^{*} B$ polymorphisms in schizophrenic patients (Table 2).

However, when we considered the combined genotypes of the four genes (Table 2), a significant increase in schizophrenia risk was found for the association between the absence of the GSTM1 gene and the presence of the GSTT1 gene $(\mathrm{OR}=1.94 ; 95 \%$ CI 1.12-3.34; power 0.70). The risk was also higher when the GSTM1 null polymorphism was combined with the GSTA1 ${ }^{*} B /{ }^{*} B$ genotype (OR $=2.6$; $95 \%$ CI 1.09 6.32; power 0.64). Finally, the risk further increased when we took into consideration the combination of the absence of the GSTM1 gene, with the presence of GSTT1 and the polymorphism GSTA1 ${ }^{*} \mathrm{~B} /{ }^{*} \mathrm{~B}$ $(\mathrm{OR}=2.8$; 95\% CI 1.10-7.23; power 0.60).

The age of onset, the baseline SANS and SAPS scores and olanzapineequivalent dosages were analyzed by ANOVA considering GST polymorphisms. No polymorphism or their combinations affected these parameters (data not shown).

\section{Discussion}

A reduced antioxidant capacity has been described in schizophrenia (Yao et al., 1998), suggesting that an accumulation of the oxidative products catecholamine $o$-quinones could lead to the degeneration of dopaminergic neurons (Smythies, 1997). GSTs, in particular the members of the GSTM class, are involved in the detoxification of oxidized metabolites (o-quinones) of catecholamines playing an antioxidant function (Baez et al., 1997; Segura-Aguilar et al., 1997; Smythies and Galzigna, 1998).

We found that the absence of the GSTM1 gene, together with the polymorphism GSTA1 ${ }^{*} B /{ }^{*} B$, leading to decreased levels of GSTA1 protein, confers a 2.6 higher risk to develop schizophrenia.

Table 2

Combination of polymorphisms in GST genes and evaluation of schizophrenia risk

\begin{tabular}{|c|c|c|c|c|c|}
\hline GST polymorphisms & Schizophrenic patients $N(\%)$ & Healthy comparison $N(\%)$ & OR & $95 \% \mathrm{CI}$ & $p$ value \\
\hline GSTM1 & & & & & 0.273 \\
\hline wt & $56(40.6)$ & $63(47.4)$ & & & \\
\hline null & $82(59.4)$ & $70(52.6)$ & & & \\
\hline GSTT1 & & & & & 0.370 \\
\hline wt & $113(81.9)$ & $103(77.4)$ & & & \\
\hline null & $25(18.1)$ & $30(22.6)$ & & & \\
\hline GSTA1 & & & & & 0.362 \\
\hline $\mathrm{A} / \mathrm{A}$ & $37(26.8)$ & $38(28.6)$ & & & \\
\hline $\mathrm{A} / \mathrm{B}$ & $71(51.5)$ & $75(56.4)$ & & & \\
\hline $\mathrm{B} / \mathrm{B}$ & $30(21.7)$ & $20(15.0)$ & & & \\
\hline GSTA1*A vs non *A & & & & & 0.343 \\
\hline GSTP1 & & & & & 0.517 \\
\hline $\mathrm{A} / \mathrm{A}$ & $66(47.9)$ & $65(48.9)$ & & & \\
\hline $\mathrm{A} / \mathrm{B}$ & $50(36.2)$ & $48(36.1)$ & & & \\
\hline $\mathrm{B} / \mathrm{B}$ & $8(5.8)$ & $9(6.8)$ & & & \\
\hline $\mathrm{A} / \mathrm{C}$ & $7(5.1)$ & $10(7.5)$ & & & \\
\hline $\mathrm{B} / \mathrm{C}$ & $5(3.6)$ & $1(0.8)$ & & & \\
\hline $\mathrm{C} / \mathrm{C}$ & $1(0.7)$ & 0 & & & \\
\hline $\mathrm{A} / \mathrm{D}$ & $1(0.7)$ & 0 & & & \\
\hline GSTP1*A vs non *A & & & & & 0.708 \\
\hline GSTP1 1 B vs non *B & & & & & 0.922 \\
\hline GSTP $1{ }^{*} \mathrm{C}$ vs non ${ }^{*} \mathrm{C}$ & & & & & 0.684 \\
\hline GSTP1*D vs non *D & & & & & 1 \\
\hline GSTM1 +/GSTT1+ & $41(29.7)$ & $54(40.6)$ & 1 & - & \\
\hline GSTM1 null/GSTT1+ & $72(52.2)$ & $49(36.8)$ & 1.935 & $1.123-3.336$ & $0.017^{*}$ \\
\hline $\mathrm{GSTM} 1+/ \mathrm{GSTA} 1 * \mathrm{~A} /{ }^{*} \mathrm{~A}$ & $15(10.9)$ & $19(14.3)$ & 1 & - & \\
\hline GSTM1 null/GSTA1 ${ }^{*} \mathrm{~B} /{ }^{*} \mathrm{~B}$ & $20(14.5)$ & $8(6)$ & 2.628 & $1.094-6.315$ & $0.031^{*}$ \\
\hline GSTM1+/GSTA1 *A/*A/GSTT1 null & $9(6.5)$ & $17(12.8)$ & 1 & - & \\
\hline GSTM1 null/GSTA1 ${ }^{*} \mathrm{~B} /{ }^{*} \mathrm{~B} / \mathrm{GSTT} 1+$ & $18(13)$ & $7(5.3)$ & 2.824 & $1.104-7.276$ & $0.030^{*}$ \\
\hline
\end{tabular}


Also GSTA1 has catalytic activity on 0 -quinones, although to a lesser extent (Baez et al., 1997). So, it is conceivable that the concomitant absence of GSTM1 and the decreased levels of GSTA1 could reduce the capacity to detoxify 0 -quinones, representing a danger for the survival of dopaminergic neurons.

GSTP1-1 is another GST that has catalytic activity on 0 -quinones (Baez et al., 1997). However, we did not find any association between GSTP1 polymorphisms and schizophrenia, probably because GSTP1 polymorphisms do not affect protein levels, but modulate GSTP1 affinity to its substrates. In accordance to our findings, it has been previously reported that GSTP1 polymorphisms do not confer susceptibility to schizophrenia (Pae et al., 2003).

Very interestingly, we found that the presence of GSTT1 increases the risk to have schizophrenia for GSTM1 null/GSTA1 ${ }^{*} B /{ }^{*} B$ carriers. GSTT1 gene maps on region 22q11.2 (Webb et al., 1996), which was found to be involved in susceptibility to schizophrenia by genomewide scan analysis (Mowry et al., 2004). Moreover, an association between the presence of the GSTT1 gene and the risk of developing schizophrenia has been recently reported (Saadat et al., 2007).

An explanation of why GSTT1 is a factor risk for schizophrenia, could be provided by recent in vivo and post-mortem studies that revealed a reduction of GSH levels in cerebrospinal fluid and in different brain regions of schizophrenic patients (Do et al., 2000; Yao et al., 2006). In this context, since GSTT1 is not involved in the detoxification of o-quinones (Baez et al., 1997), its antioxidative action towards other substrates might induce a further decrease in glutathione levels. In this context, the capacity to use GSH to detoxify $o$-quinones would be decreased by the combination of low GSH levels, the absence of GSTM1, the lower levels of GSTA1 and the presence of GSTT1, which consumes GSH to detoxify other substrates.

Some limitations of the study need to be mentioned. Firstly, we cannot exclude the possibility that the control participants in our study will develop schizophrenia, even though control participants under 30 years old were only the $9.8 \%$ of the sample. Secondly, we performed a series of ANOVAs to investigate if positive and negative symptomatology (assessed with the PANSS positive and negative scales) and olanzapine equivalents may be related to GST polymorphisms. We did not find differences for such variables (data available upon request); however, further research is needed to clarify this issue.

In conclusion, we found that the combination of polymorphisms in GST genes represents a risk factor for schizophrenia. Moreover, these data strengthen the hypothesis of a role for the detoxification potential against oxidative stress, and in particular against 0 -quinones detoxification, in the development of schizophrenia.

\section{References}

American Psychiatric Association, 2004. Diagnostic and Statistical Manual of Mental Disorders. APA, Washington, DC.

Baez, S., Segura-Aguilar, J., Widersten, M., Johansson, A.S., Mannervik, B., 1997. Glutathione transferases catalyse the detoxication of oxidized metabolites (o-quinones) of catecholamines and may serve as an antioxidant system preventing degenerative cellular processes. The Biochemical Journal 324, 25-28.
Bernardini, S., Bellincampi, L., Ballerini, S., Federici, G., Iori, R., Trequattrini, A., Ciappi, F. Baldinetti, F., Bossu, P., Caltagirone, C., Spalletta, G., 2005. Glutathione S-transferase P1 ${ }^{*} \mathrm{C}$ allelic variant increases susceptibility for late-onset Alzheimer disease: association study and relationship with apolipoprotein E epsilon4 allele. Clinical Chemistry 51, 944-951.

Cornelis, M.C., El-Sohemy, A., Campos, H., 2007. GSTT1 genotype modifies the association between cruciferous vegetable intake and the risk of myocardial infarction. The American Journal of Clinical Nutrition 86, 752-758.

Do, K.Q., Trabesinger, A.H., Kirsten-Kruger, M., Lauer, C.J., Dydak, U., Hell, D., Holsboer, F., Boesiger, P., Cuenod, M., 2000. Schizophrenia: glutathione deficit in cerebrospinal fluid and prefrontal cortex in vivo. The European Journal of Neuroscience 12, 3721-3728.

Freedman, R., 2003. Schizophrenia. The New England Journal of Medicine 349, 1738-1749.

Gaziev, J., Nguyen, L., Puozzo, C., Mozzi, A.F., Casella, M., Perrone Donnorso, M., Gravina, P., Sodani, P., Marziali, M., Isgrò, A., Simone, M.D., Andreani, M., Formosa, A., Testi, M., Federici, G., Bernardini, S., Lucarelli, G., 2010. Novel pharmacokinetic behavior of intravenous busulfan in children with thalassemia undergoing hematopoietic stem cell transplantation: a prospective evaluation of pharmacokinetic and pharmacodynamic profile with therapeutic drug monitoring. Blood 115, 4597-4604.

Kay, S.R., Fiszbein, A., Opler, L.A., 1987. The Positive and Negative Syndrome Scale (PANSS) for schizophrenia. Schizophrenia Bulletin 13, 261-276.

Marini, A., Spoletini, I., Rubino, I.A., Ciuffa, M., Bria, P., Martinotti, G., Banfi, G., Boccascino, R., Strom, P., Siracusano, A., Caltagirone, C., Spalletta, G., 2008. The language of schizophrenia: an analysis of micro and macrolinguistic abilities and their neuropsychological correlates. Schizophrenia Research 105, 144-155.

Mowry, B.J., Holmans, P.A., Pulver, A.E., Gejman, P.V., Riley, B., Williams, N.M., Laurent C., Schwab, S.G., Wildenauer, D.B., Bauche, S., Owen, M.J., Wormley, B., Sanders, A.R., Nestadt, G., Liang, K.Y., Duan, J., Ribble, R., Norton, N., Soubigou, S., Maier, W., EwenWhite, K.R., DeMarchi, N., Carpenter, B., Walsh, D., Williams, H., Jay, M., Albus, M. Nertney, D.A., Papadimitriou, G., O'Neill, A., O'Donovan, M.C., Deleuze, J.F., Lerer, F.B., Dikeos, D., Kendler, K.S., Mallet, J., Silverman, J.M., Crowe, R.R., Levinson, D.F., 2004. Multicenter linkage study of schizophrenia loci on chromosome 22q. Molecular Psychiatry 9, 784-795.

Oquendo, M.A. Baca-Garcia, E., Kartachov, A., Khait, V., Campbell, C.E., Richards, M. Sackeim, H.A., Prudic, J., Mann, J.J., 2003. A computer algorithm for calculating the adequacy of antidepressant treatment in unipolar and bipolar depression. The Journal of Clinical Psychiatry 64, 825-833.

Pae, C.U., Kim, J.J., Lee, S.J., Lee, C.U., Lee, C., Paik, I.H., Park, H.R., Yang, S., Serretti, A 2003. Association study between glutathione $S$-transferase P1 polymorphism and schizophrenia in the Korean population. Progress in Neuro-psychopharmacology \& Biological Psychiatry 27, 519-523.

Saadat, M., Mobayen, F., Farrashbandi, H., 2007. Genetic polymorphism of glutathione $S$-transferase T1: a candidate genetic modifier of individual susceptibility to schizophrenia. Psychiatry Research 153, 87-91.

Segura-Aguilar, J., Baez, S., Widersten, M., Welch, C.J., Mannervik, B., 1997. Human class Mu glutathione transferases, in particular isoenzyme M2-2, catalyze detoxification of the dopamine metabolite aminochrome. The Journal of Biological Chemistry 272 5727-5731.

Smythies, J.R., 1997. Oxidative reactions and schizophrenia: a review-discussion. Schizophrenia Research 24, 357-364.

Smythies, J., Galzigna, L., 1998. The oxidative metabolism of catecholamines in the brain: a review. Biochimica et Biophysica Acta 1380, 159-162.

Spoletini, I., Cherubini, A., Di Paola, M., Banfi, G., Rusch, N., Martinotti, G., Bria, P., Rubino I.A., Siracusano, A., Caltagirone, C., Spalletta, G., 2008. Reduced fronto-temporal connectivity is associated with frontal gray matter density reduction and neuropsychological deficit in schizophrenia. Schizophrenia Research 17, 17.

Valentini, A., Gravina, P., Bernardini, S., Federici, G., 2008. Role of glutathione Stransferase in the cellular antioxidant defence. In: Eleuteri, A.M. (Ed.), Enzymes and the Cellular Fight Against Oxidation. Research Signpost, Kerala, India.

Webb, G., Vaska, V., Coggan, M., Board, P., 1996. Chromosomal localization of the gene for the human theta class glutathione transferase (GSTT1). Genomics 33, 121-123.

Yao, J.K., Reddy, R., McElhinny, L.G., van Kammen, D.P., 1998. Reduced status of plasma total antioxidant capacity in schizophrenia. Schizophrenia Research 32, 1-8.

Yao, J.K., Leonard, S., Reddy, R., 2006. Altered glutathione redox state in schizophrenia. Disease Markers 22, 83-93. 